\title{
Mitotic Processes which Restore Genome Balance in Aspergillus nidulans
}

\author{
By B. L. CASE AND J. A. ROPER* \\ Department of Genetics, The University, Sheffield S10 2TN
}

(Received 17 September 1980)

\begin{abstract}
Previous work had shown that haploid strains of Aspergillus nidulans with a duplicate chromosome segment (one in normal position, one translocated to another chromosome) were unstable at mitosis; genome balance was restored by spontaneous deletion of either duplicate segment. Diploids with an extra, translocated segment showed high instability which was confined to the excess segments; loss of one of these, usually that in translocated position, gave balanced diploid nuclei and the loss was assumed to be by deletion. This led to the proposal that high-frequency deletion was provoked by, and confined to, the excess segment. In the present work it has been shown that elimination of the translocated segment in such diploids occurs more frequently by mitotic crossing over than by deletion. Accordingly, in a more rigorous test of the possible association of excess segments and deletions, a diploid homozygous for an extra, translocated segment has been studied as mitotic crossing over in this strain could not give a balanced genome. The strain was extremely unstable and gave variants of which most had a balanced, or near-balanced, diploid genome. Some variants arose by simultaneous deletions involving both non-translocated segments; almost all variants had deletions with breakpoints different from those most frequent in the corresponding, duplication haploid. The results have shown the diversity of mechanisms available for the correction of genome imbalance and that, at least in the case of $\mathrm{Dp}(\mathrm{I}, \mathrm{II})$, the degree and modalities of mitotic instability are functions of the balance of chromosome segments and of ploidy.
\end{abstract}

\section{INTRODUCTION}

Because of its colonial growth and uninucleate conidia with autonomous colour mutants, Aspergillus nidulans has been an amenable species for the study of 'errors' of mitosis. These properties facilitated isolation of strains with heterozygous, diploid nuclei and demonstration of the mitotic crossing over, non-disjunction and haploidization that diploid nuclei undergo (for review, see Roper, 1966).

Another type of mitotic error was discovered in haploids which had a chromosome segment in duplicate [Dp(III,VIII) and Dp(I,II); Fig. 1]. Genetic imbalance caused slow growth of these strains but they produced faster-growing sectors, less unbalanced than the parent, by spontaneous deletion of part or all of either duplicate segment (Bainbridge \& Roper, 1966; Nga \& Roper, 1968). Nga \& Roper (1969) appeared to have found evidence of a causal relationship between duplications and deletions when they studied the unbalanced diploid $\mathrm{Dp}(\mathrm{I}, \mathrm{II}) / / \mathrm{MSE}$ (Fig. 1). Very high instability in this strain was confined to the triplicate segments; loss of a segment, usually that in translocated position, was assumed always to be by deletion. It was suggested that segments present in excess tend to suffer replication errors resulting in loss of one of them and restoration of genome balance.

In the present work on similar diploids, but using more genetic markers, it has been shown that genome balance can be restored by two independent processes - deletion and mitotic crossing over. Attempts have been made, therefore, to determine the relative contributions of 
the two processes to the total instability. In addition, a diploid homozygous for an extra, translocated, chromosome segment has been studied; only deletion, not mitotic crossing over, could restore genome balance in this and the strain provided a more rigorous test of the proposed association between imbalance and deletions.

\section{METHODS}

Media. Minimal medium was Czapek-Dox with $1 \%(\mathrm{w} / \mathrm{v})$ glucose. Complete medium contained yeast extract, hydrolysed casein, hydrolysed nucleic acids, vitamins, etc.

Methods of analysis. General methods were those of Pontecorvo et al. (1953). More specialized methods have been detailed by Nga \& Roper (1968). Incubation was at $37^{\circ} \mathrm{C}$.

Estimates of instability. Counts of sectors and of patches of mycelium showing the products of instability were made on colonies each grown from a single conidium.

Strains. All were derived from Glasgow stocks. Master strains E and F (MSE and MSF), with markers on all linkage groups, were those of McCully \& Forbes (1965). The principal mutant alleles used, and the phenotypes determined, are listed below using Clutterbuck's $(1970,1974)$ designations: $w A 3, w A 7, y g A 6$, and $y A 2-$ white, pale, yellow-green, and yellow conidia, respectively; abA1, adE20, biA1, nicB8, pabaA6, proA1, pyroA4, riboA1, riboB 2 and riboE6, $s A 1$ and $s C 12$, and thiA4 - requirement for, respectively, $\alpha$-aminobutyric acid, adenine, biotin, nicotinic acid, $p$-aminobenzoic acid, proline, pyridoxine, riboflavin, thiosulphate, and thiazole; $c n x E 16, f a c A 303$, and galA $I$ - inability to use nitrate, acetate, and galactose, respectively; $\operatorname{acr} A I$ and $\operatorname{acr} B 2$ - resistance to acriflavine; sUA ladE20 - suppressor of adE20.

Duplication strains; symbols. Figures 1, 2 and 3 show the principal duplication strains used. The conventions adopted to give genotypes in the text are shown by the following example: Dp(I,II) pabaA yA $a d E^{+} b i A^{+} /$ $y A^{+} a d E b i A ; w A 7$ thiA designates the I,II duplication strain carrying pabaA on the unduplicated part of chromosome I, $y A, a d E^{+}$and $b i A^{+}$on the untranslocated segment of IR, $y A^{+}, a d E$ and $b i A$ on the homologous, translocated segment, and $W A 7$ and thiA in a different linkage group. For simplicity, allele numbers are excluded from the text and figures except when, as in the case of $w A 3$ and $w A 7$, it is essential to distinguish different mutant alleles of one gene. The haploid components of diploid strains are separated by the symbol $/ /$.

\section{RES ULTS}

\section{Diploids heterozygous for an extra chromosome segment}

Sectors of strain A (Fig. 2) indicated that a process other than deletion had produced some balanced diploids from an unbalanced diploid. The 29 colonies examined had 145 clear-cut

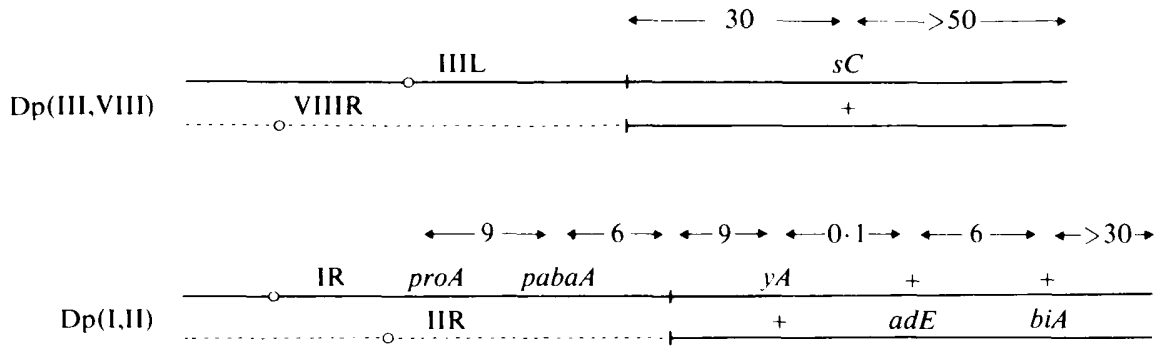

\begin{tabular}{|c|c|c|c|c|c|c|}
\hline & + & proA & pabaA & $y A$ & + & \pm \\
\hline & suAadE & + & + & $y A$ & $a d E$ & + \\
\hline $\mathrm{Dp}(\mathrm{I}, \mathrm{II}) / / \mathrm{MSE}$ & & & & + & $a d E$ & $b i A$ \\
\hline
\end{tabular}

Fig. 1. The basic constitution of some strains used in the first studies of duplication strain instability. To define the duplications, some loci and approximate meiotic recombination frequencies are shown. The different chromosomes in each duplication are distinguished by broken and unbroken lines: centromeres are shown by circles. Duplication strains (I,II) and (III,VIII) carry terminal segments of IR and IIIL attached terminally and uninverted to IIR and VIIIR respectively. Diploid Dp(I,II)//MSE was heterozygous for genes on all eight linkage groups. See Methods for definition of symbols. 

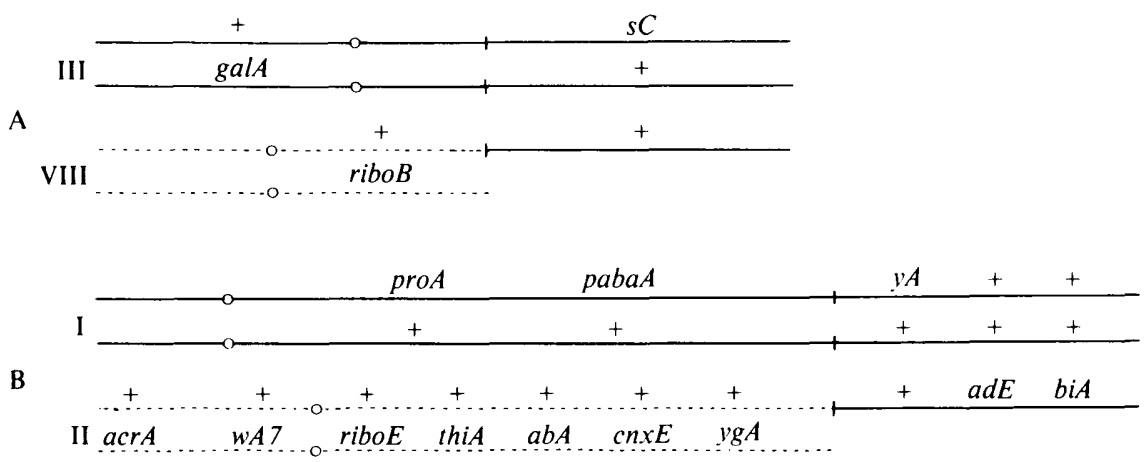

Fig. 2. Duplication diploids used to investigate mitotic crossing over as a means of restoring a balanced genome. The different chromosomes in each duplication are distinguished by broken and unbroken lines; centromeres are shown by circles. Only markers necessary to establish the essential findings are shown. Strain A was heterozygous also for biA and the markers of MSF.

sectors after $6 \mathrm{~d}$ incubation. Of these sectors 67 were stable haploids with various combinations of the mutant alleles for which the parent was heterozygous. The rest were stable diploids, 60 prototrophs and 18 riboflavin-requiring. The unexpected, riboflavinrequiring sectors could have arisen by either of two processes. Non-disjunction of chromosome VIII could have eliminated the translocated segment and given a product homozygous for riboB (Pontecorvo \& Käfer, 1958). Alternatively, mitotic crossing over between the locus of $r i b o B$ and its centromere, and appropriate strand segregation, could have achieved the same result. The origin of the 60 green prototrophs had also to be questioned. An unknown proportion of these could have arisen by crossing over with the exchange distal to riboB; the resulting sectors, still heterozygous for $r i b o B$, would have been indistinguishable from those which arose by deletion of the whole translocated segment.

Strain B (Fig. 2) was used to estimate the contribution of mitotic crossing over and/or non-disjunction to the total instability of a $\mathrm{Dp}(\mathrm{I}, \mathrm{II})$ diploid. The 94 colonies examined gave 920 sectors (Table 1). The few yellow haploids (class A) resulted from deletion and haploidization, as simultaneous or successive events. Balanced green haploids $\left(\mathrm{pro}^{+} \mathrm{paba}^{+} \mathrm{y}^{+} ; \mathrm{w}^{+}\right.$) of similar origin were not found, but this was probably due to failure to detect them against the parental, green background. Pale yellow and pale green haploids (classes B and C) were consistent with haploidization; as usual, haploids with a duplicate segment were not obtained as there is severe selection against them. A large sample of diploid

\section{Table 1. Sectors from unbalanced diploid $B$}

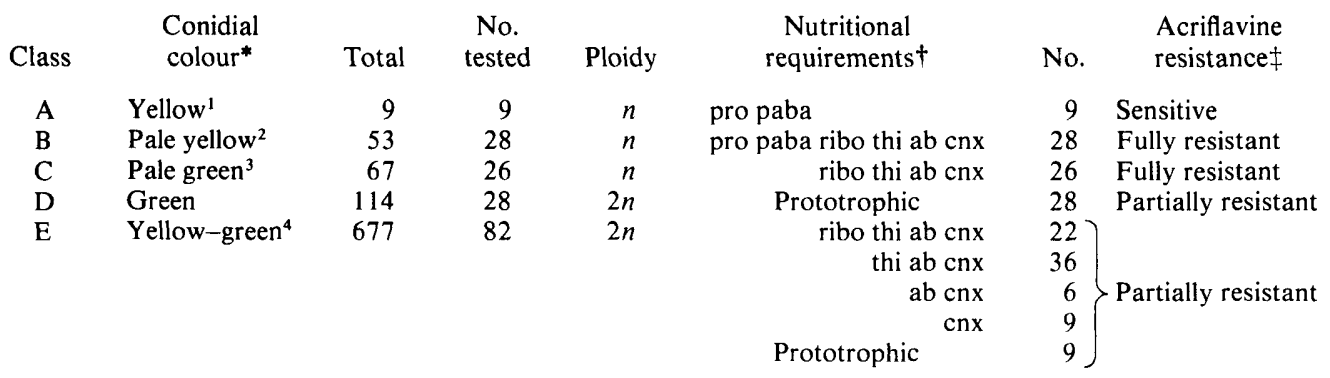

* Genotypes for conidial colour markers: (1) $y A: w A 7^{+} y g A^{+}$. (2) $y A ; w A 7 y g A$. (3) $y A^{+} ; w A 7 y g A$. (4) Homozygous $y g A$ : a sample of these were shown, by further segregation, all to be heterozygous $y A / y A^{+} ; w A 7 / w A 7^{+}$.

+ Symbols for nutritional requirements are defined as for the corresponding genotype symbols.

$\ddagger$ acr $A$ is semi-dominant and partial resistants are heterozygous. 
sectors (classes D and E) were tested and none showed greater instability than that expected of a balanced diploid. The yellow-green diploids clearly arose by mitotic crossing over between the $y g A$ locus and its centromere and the subsequent segregation of strands eliminated the translocated segment; 82 class $E$ sectors were tested for nutritional requirements and showed that there had been exchanges in all marked intervals of IIR. The green prototrophic diploids (class D) could not have arisen by non-disjunction as they had the genotype $\mathrm{acr} / \mathrm{acr}^{+}$; non-disjunction produces nuclei homozygous for all the markers on the chromosome concerned. A total of 74 were haploidized to determine which of the triplicate segments had been lost. Three gave only two classes of balanced haploids: green pro paba ad bi and pale green acr ribo thi ab cnx; these three sectors had arisen by deletion of the segment carrying $y \mathrm{~A} \mathrm{adE^{+ }} \mathrm{biA}^{+}$. The remaining 71 gave four classes of balanced haploids which combined either homologue of chromosome I with either of chromosome II; these had lost the translocated segment either by deletion or by mitotic crossing over distal to $y g A$. Of 791 balanced diploid sectors from strain B, less than the 114 in class D could have arisen by deletion; that is, the maximum frequency of detected deletions per colony was within the range found in haploid Dp(I,II) strains (Nga \& Roper, 1968, 1969). Diploid B had revealed novel details about the elimination of excess genetic material but the overall results neither refuted nor added weight to the proposed causal relationship between imbalance and deletions.

\section{Diploids homozygous for an extra chromosome segment}

The duplication haploids used to synthesize strain $\mathrm{C}$ (Fig. 3) were $\mathrm{Dp}(\mathrm{I}, \mathrm{II})$ riboA $y A$ adE $E^{+}$biA $/ y A$ adE $E^{+} b i A^{+} ; n i c B$ and $\mathrm{Dp}(\mathrm{I}, \mathrm{II}) y A$ adE $E^{+}$biA $/ y A^{+}$adE biA; thiA;sA; pyroA. The instability of each haploid component was tested and its breakdown products were analysed meiotically and via the parasexual cycle. Each component was combined with a standard haploid and the resulting diploids, as well as their breakdown products, were analysed. All tests confirmed the stated genotypes of the duplication haploids, which were then com-

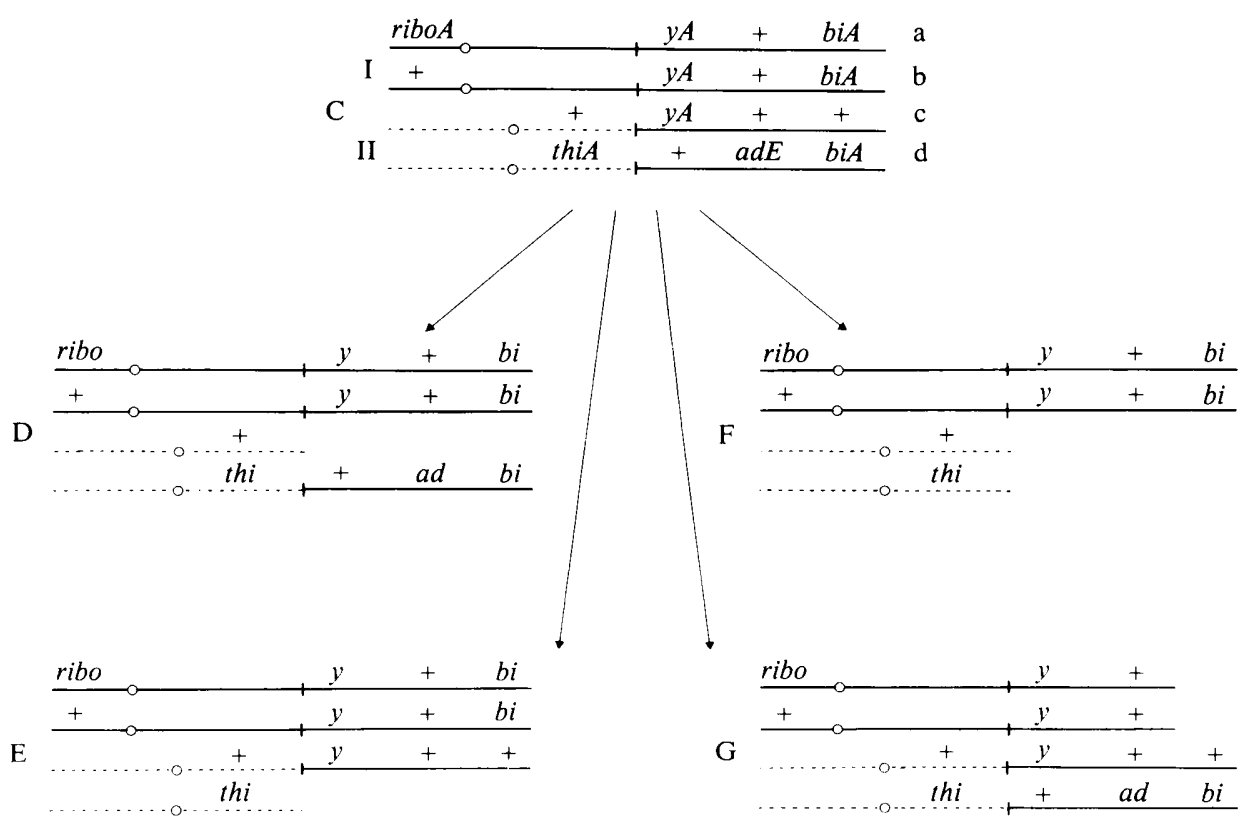

Fig. 3. Strain C, homozygous for $\mathrm{Dp}(\mathrm{I}, \mathrm{II})$. In addition to the markers shown it was heterozygous for $s A$ (III), pvroA (IV) and nicB (VII). For reference the segments present in quadruplicate are lettered a to d. Arrows indicate the detected deletion products D, E, F and $\mathrm{G}$ in which, for simplicity, locus letters are excluded. 
Table 2. Sectors from strain $C$

\begin{tabular}{|c|c|c|c|c|c|c|}
\hline $\begin{array}{l}\text { Conidial } \\
\text { colour }\end{array}$ & Total* & $\begin{array}{l}\text { No. } \\
\text { tested }\end{array}$ & Ploidy & Phenotype & No. & Class \\
\hline Green & 763 & 329 & $\begin{array}{l}2 n \\
2 n\end{array}$ & $\begin{array}{l}\text { Prototrophic } \\
\text { Biotin requiring }\end{array}$ & $\begin{array}{r}305 \\
24\end{array}$ & $\begin{array}{l}1 \\
2\end{array}$ \\
\hline Yellow & 70 & 60 & $\begin{array}{l}2 n \\
2 n\end{array}$ & $\begin{array}{l}\text { Prototrophic } \\
\text { Biotin requiring }\end{array}$ & $\begin{array}{r}57 \\
3\end{array}$ & $\begin{array}{l}3 \\
4\end{array}$ \\
\hline
\end{tabular}

bined in duplicate heterokaryons to give two independent isolates of C. Haploidization of both isolates gave similar results in which the parental, recessive markers were recovered; the isolates also gave similar instability products and results with them were therefore pooled.

Strain $C$ produced an average of about 10 clear-cut sectors per colony (Table 2). Of the sectors tested, 6 green and 10 yellow sectors (some haploid, some diploid) have been excluded from Table 2. They were auxotrophic for various markers and probably resulted from combinations of mitotic crossing over, non-disjunction, deletion and/or haploidization. As minority classes they would not have revealed the main processes of instability.

Classes 2 and 3 (Table 2), presumably resulting from deletion of segments $c$ and $d$ respectively, had been expected as the majority class as over $60 \%$ of the deletions in Dp(I,II) haploids involve the translocated segment. One isolate of each class, assumed to be typical, was analysed by study of its own instability and by genetic analysis of haploids derived from it. These examples of classes 2 and 3 were shown to have the constitutions $D$ and $E$, respectively (Fig. 3). Class 2 sectors may have been underscored because of their parental colour. Even with allowance for this, sectors arising by deletion of either translocated segment in strain $\mathrm{C}$ (about two per colony) were probably not significantly more than those arising by deletion of the translocated segment in Dp(I,II) haploids.

The three yellow, biotin-requiring sectors (class 4) were stable on complete medium. One was haploidized and, through recovery of all markers except those on the translocated segments of strain $\mathrm{C}$, was shown to be type F (Fig. 3). This sector, phenotypically typical of class 4 , was a standard, balanced diploid which arose by loss of segments $\mathrm{c}$ and $\mathrm{d}$.

About $80 \%$ of the strain $\mathrm{C}$ sectors were green, prototrophic diploids. A random sample of 15 were judged to have balanced or near-balanced genomes on the bases of growth rate, morphology and stability; they must have resulted from loss roughly equivalent to two of the segments originally present in quadruplicate. They retained $y^{+}$(green) and $\mathrm{bi}^{+}$(biotin independence) so the deletions must have involved any combination of the untranslocated segments ( $\mathrm{a}$ and $\mathrm{b})$ and the translocated segments distal to $b i^{+}(\mathrm{c})$ and $y^{+}(\mathrm{d})$. Despite their phenotypic similarity the 15 isolates were genetically heterogeneous. Some were completely resistant to haploidization, others yielded only aconidial, putative haploids; partial analysis was achieved in six cases but definitive analysis in only two. Total characterization depended on haploidization, to reveal recessive alleles, and crosses of the haploids to determine deletion breakpoints.

Haploids from 6 of the 15 prototrophic sectors showed that they all carried the mutant alleles $y, b i$, nic, pyro, ribo, $s$ and thi. Adenine-requiring haploids were not obtained but, as shown below, this was not proof of the absence of the mutant ad allele. Yellow haploids were obtained from sectors 8,5 and 15 , some expressing ribo, others expressing ribo $^{+}$; that is, the two chromosome I homologues of these sectors had been recovered in haploids with various combinations of the mutant and wild-type alleles on chromosome III $(s A)$, IV (pyroA) and VII (nicB). One ribo ${ }^{+}$and one ribo haploid from each sector was combined with a standard haploid, proA1 biA1 (I); wA3 thiA4 acrB2 (II); pyroA4 (IV); facA303 (V), and the resulting six diploids were haploidized. Between 8 and 20 white haploids were obtained from each 
Table 3. Crosses of haploids derived from strain $C$ sectors

\begin{tabular}{|c|c|c|c|c|c|c|}
\hline \multirow[b]{3}{*}{ Parents* } & \multicolumn{2}{|c|}{ Morphological classification } & \multicolumn{4}{|c|}{ Nutritional classification } \\
\hline & & 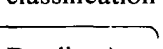 & \multicolumn{2}{|c|}{ Duplication progeny ${ }^{\dagger}$} & \multicolumn{2}{|c|}{ Non-duplication progeny } \\
\hline & Normal & type & paba $^{+}$ & paba & $\mathrm{bi}^{+}$ & bi \\
\hline $22 \times 5 a$ & 298 & 141 & 49 & 251 & 86 & 0 \\
\hline $22 \times 5 b$ & 292 & 124 & 70 & 223 & 387 & 0 \\
\hline $22 \times 8 a$ & 460 & $171 \neq$ & 15 & 38 & 56 & 0 \\
\hline $22 \times 8 b$ & 463 & 215 & 23 & 89 & 29 & 0 \\
\hline
\end{tabular}

Several separate ascospore platings were made for each cross; this accounts for the different numbers of progeny used in morphological and nutritional classifications.

* Strain 22, a standard haploid, had the genotype proAl pabaA6 vA2; thiA4; facA303; riboB2. The other parents were haploids derived from green, prototrophic, diploid sectors of strain $C$. They expressed the following mutant alleles: 5a - yA2 riboA1 pyroA4 nicB8; 5 b - yA2 pyroA4 nicB8; 8 a - yA2 riboA1 pyroA4 sA1 nicB8; $8 \mathrm{~b}-y A 2$ pyroA $4 \mathrm{sA} 1$ nicB 8 .

+ On the basis of this classification the lethal mutations, representing the proximal points of deletion, were mapped distal to the pabaA locus with the following approximate recombination frequencies: $5 \mathrm{a}, 16 \% ; 5 \mathrm{~b}, 24 \%$; $8 \mathrm{a}, 28 \% ; 8 \mathrm{~b}, 21 \%$.

$¥$ In this case significantly less than one-third of the total progeny were of duplication type; this is a fairly common experience attributed partly to lower viability of this class and to their misclassification on crowded plates.

diploid and all required proline. That is, both chromosome I homologues of isolates 5, 8 and 15, marked by pro $^{+}$ribo $^{+}$and pro $^{+}$ribo, had recessive, effectively-lethal mutations which prevented their recovery with a standard chromosome II, although they could be obtained in haploids with either homologue of the II-I complexes of the diploid sectors 5,8 and 15 . The results indicated deletions in both chromosome I homologues of these three sectors which behaved like balanced, or near-balanced, translocation homozygotes. Sectors 5 and 8 were analysed further by crosses of their ribo and ribo $^{+}$haploids to a standard haploid, proAl pabaA6 yA2 (I); thiA4 (II); facA303 (V); riboB2 (VIII). All four crosses (Table 3) gave an approximately $2: 1$ ratio of balanced to duplication progeny which was consistent with the tested strains being balanced haploids with a I,II translocation (Bainbridge \& Roper, 1966; Nga \& Roper, 1968). The proximal breakpoints of the chromosome I deletions in the tested strains were determined by classifying duplication progeny for $p$-aminobenzoic acid requirement; the $\mathrm{paba}^{+}$class, with an intact chromosome I, arose by crossing over between the proximal breakpoint and the paba locus on the partially deleted chromosome I. Uncertainties about chromosome pairing in a translocation heterozygote did not permit accurate location but the proximal breakpoints -16 to 28 units distal to $p a b a$ - were probably near the $b i$ locus. This would have left the $a d^{+}$alleles on chromosome I and so explained the absence of adenine-requiring haploids from the tested, green, prototrophic sectors. Whether the breakpoints were distal or proximal to $b i$ was determined as follows. If the mutant $b i$ allele was still present on chromosome I of the yellow haploids $5 \mathrm{a}, 5 \mathrm{~b}, 8 \mathrm{a}$ and $8 \mathrm{~b}$ (but not expressed because of $\mathrm{bi}^{+}$on the translocated segment) then outcrossing should have given some recombinant, non-duplication bi progeny. None was found (Table 3). In sectors 5 and 8 the proximal breakpoints on both chromosome I homologues were between the ad and bi loci (type G, Fig. 3).

\section{DIS CUSSION}

The overall pattern of spontaneous mitotic instability in haploid and diploid strains with the (I,II) duplication is complex; the major genetic consequences of instability are being defined, but total understanding of the mechanisms is not in sight. $\mathrm{Dp}(\mathrm{I}, \mathrm{II})$ haploids suffer deletions, mainly from the duplicate segment in translocated position. Diploids heterozygous for $\mathrm{Dp}(\mathrm{I}, \mathrm{II})$ undergo deletions but most of the instability restoring genome balance is due to 
elimination of the translocated segment by mitotic crossing over. Diploids homozygous for $\mathrm{Dp}(\mathrm{I}, \mathrm{II})$ show extreme instability for the segments present in quadruplicate and deletions, not mitotic crossing over, are responsible; although only few variants of this diploid have been analysed fully, it is clear that deletions occur mainly in positions different from those which are frequent in the duplicate segments of $\mathrm{Dp}(\mathrm{I}, \mathrm{II})$ haploids.

High instability of the quadruplicate segments in $\mathrm{Dp}(\mathrm{I}, \mathrm{II})$ homozygotes supports the idea that excess segments provoke their own instability through a process which, whatever its details, is effectively intrachromosomal (Nga \& Roper, 1969). Majerfeld \& Roper's (1978) study of the effects of coumarin on Dp(I,II) haploids gives indirect support to the association between imbalance and deletions and suggests some detail of the mechanism. Coumarin increased the deletion frequency by selective action on the translocated segment; the results suggested that the attachment region of the translocated segment generates spontaneous, recombinogenic lesions which, if not repaired, lead to deletion. A balanced translocation (I,II) haploid, with the attachment region but no duplicate segment, gave no evidence of such recombinogenic lesions. The effects of ploidy on deletion breakpoints, and presumably on the DNA lesions from which they are generated, is emphasized by the action of coumarin which, at concentrations highly effective in $\mathrm{Dp}(\mathrm{I}, \mathrm{II})$ haploids, did not significantly increase the instability of the Dp(I,II) homozygote used in this work (Relton \& Roper, unpublished). Newmeyer \& Galeazzi (1977) and Birkett \& Roper (1977) have suggested mechanisms which are similar to each other to explain mitotic deletions; although these mechanisms are more precisely defined than that of Nga \& Roper (1969), the ideas may not be mutually exclusive. To explain the exact and usually preferential deletion of the terminal, translocated segment in some Neurospora crassa duplication strains, Newmeyer \& Galeazzi (1977) proposed crossing over within an intrachromosomal loop formed by pairing between the telomere sequence of the translocated segment and a similar, residual sequence at its attachment point. Birkett \& Roper (1977) proposed a more general model involving pairing of the telomere sequence with any one of a number of similar, internal sequences; this would explain deletions from the translocated or untranslocated segments, partial deletion of a segment, and the occasional production of 'sticky ends' which might be aberrant telomeres. However, the models would need refinement to explain the effects of ploidy.

Search for an explanation of mitotic instability in duplication strains of $A$. nidulans is complicated by the fact that strains with different duplications, although all unstable, show different instability patterns; they differ in the relative frequencies of deletions between and within the duplicate segments, and in their responses to chemical and genetic agents (Birkett \& Roper, 1977; Burr \& Roper, unpublished). Understanding of the instability phenomena appears to require detailed knowledge, which does not yet exist, of the effects on mitotic fidelity of genome organization, of the balance of chromosome segments, of ploidy, and probably also of the control of DNA replication. Current studies (Sexton \& Roper, unpublished) on strains carrying a new series of spontaneous duplications may take us some way towards certain of these objectives.

The authors are indebted to Mrs J. Relton for expert help in the analyses. Financial support from the Yorkshire Council for Cancer Research is acknowledged gratefully.

\section{REFERENCES}

BAinbridge, B. W. \& Roper, J. A. (1966). Observations on the effects of a chromosome duplication in Aspergillus nidulans. Journal of General Microbiology 42, 417-424.

Birkett, J. A. \& Roper, J. A. (1977). Chromosome aberrations in Aspergillus nidulans. In The
Genetics and Phvsiologv of Aspergillus, pp. 293303. Edited by J. E. Smith \& J. A. Pateman. New York: Academic Press.

Clutterbuck, A. J. (1970). Aspergillus symbols, locus letters and allele numbers. Aspergillus Newsletter 11, 25-33. 
Clutterbuck, A. J. (1974). Aspergillus nidulans. In Handbook of Genetics, vol. 1, pp. 447-510. Edited by R. C. King. New York: Plenum Press.

MCCully, K. S. \& Forbes, E. (1965). The use of $p$-fluorophenylalanine with 'master strains' for assigning genes to linkage groups. Genetical Research 6, 352-359.

MAJERFEld, I. H. \& Roper, J. A. (1978). The effects of coumarin on the frequency of deletions in a duplication strain of Aspergillus nidulans. Molecular and General Genetics 159, 203-206.

NewmeYer, D. \& Galeazzi, D. R. (1977). The instability of Neurospora duplication $D p(I L \rightarrow I R) H 4250$, and its genetic control. Genetics 85, 461-487.

NGA, B. H. \& Roper, J. A. (1968). Quantitative intrachromosomal changes arising at mitosis in Aspergillus nidulans. Genetics 58, 193-209.
NgA, B. H. \& Roper, J. A. (1969). A system generating spontaneous intrachromosomal changes at mitosis in Aspergillus nidulans. Genetical Research 14, 63-70.

Pontecorvo, G. \& KäFer, E. (1958). Genetic analysis by means of mitotic recombination. Advances in Genetics 9, 71-104.

Pontecorvo, G., Roper, J. A., Hemmons, L. M., Macdonald, K. D. \& Bufton, A. W. J. (1953). The genetics of Aspergillus nidulans. Advances in Genetics 5, 141-238.

Roper, J. A. (1966). The parasexual cycle. In The Fungi, vol. 2, pp. 589-617. Edited by G. C. Ainsworth \& A. S. Sussman. London: Academic Press. 